\title{
ON A GENERALIZATION OF KRASNOSELSKII'S THEOREM
}

\author{
DARIUSZ IDCZAK and ANDRZEJ ROGOWSKI
}

(Received 25 August 1999 revised 24 April 2001)

Communicated by $\mathrm{K}$. Ecker

\begin{abstract}
In this paper we prove a generalization of the well known theorem of Krasnoselskii on the superposition operator in which the domain of Nemytskii's operator is a product space. We also give an application of this result.
\end{abstract}

2000 Mathematics subject classification: primary 49J50, 47H30, 47N10.

Keywords and phrases: Nemytskii's operator, variational methods, continuous differentiability.

\section{Introduction}

Let $f: \Omega \times \mathbb{R}^{l} \rightarrow \mathbb{R}^{m}, \Omega \subset \mathbb{R}^{s}$ be a Carathéodory function (that is, measurable with respect to the first variable and continuous with respect to the group of others). In nonlinear analysis, the operator of superposition (often referred to as Nemytskii's operator) of the form $F: L^{p}\left(\Omega ; \mathbb{R}^{l}\right) \ni x(\cdot) \mapsto f(\cdot, x(\cdot)) \in L^{1}\left(\Omega ; \mathbb{R}^{m}\right), p \geq 1$ (see $[5,6,7,8])$, plays an essential role. The well-known theorem of Krasnoselskii says that an operator $F$ is continuous if it is bounded, that is, $|f(t, x)| \leq a|x|^{p}+b(t)$ for some $a>0$ and $b \in L^{1}(\Omega)$. For nonlinear operators, this is quite remarkable. The original proof of this theorem and its known modifications are long and not elementary (see for example $[5,6,9])$. A very simple and short proof of the theorem of Krasnoselskii can be found in [10]. In this paper we prove a generalization of Krasnoselskii's theorem in which the domain of Nemytskii's operator is a product space. There exists an extensive list of different kinds of generalizations of the theorem of Krasnoselskii (see $[2,1]$ and the references therein) but the authors have not found the results proved in this paper anywhere. The result we obtained is used to prove some differentiability properties of integral functionals.

(C) 2002 Australian Mathematical Society $1446-7887 / 2000 \$ A 2.00+0.00$ 


\section{Some generalization of the Krasnoselskii theorem}

Recall the classical theorem of Krasnoselskii [5].

Let $\Omega \subset \mathbb{R}^{s}$ be a (Lebesgue) measurable set and let $f: \Omega \times \mathbb{R}^{l} \rightarrow \mathbb{R}^{m}, s, l, m \in \mathbb{N}$, be a Carathéodory function. It is well known that for any measurable function $x: \Omega \rightarrow \mathbb{R}^{l}$, the function $\Omega \ni t \mapsto f(t, x(t)) \in \mathbb{R}^{m}$ is measurable.

THEOREM 1 (Krasnoselskii). Let $p_{1}, p_{2} \in[1,+\infty)$. If there exist a constant $a>0$ and a function $b \in L^{p_{2}}(\Omega)$, such that $|f(t, x)| \leq a|x|^{p_{1} / p_{2}}+b(t)$ for $t \in \Omega$ a.e., $x \in \mathbb{R}^{l}$, then the Nemytskii operator $F: L^{p_{1}}\left(\Omega ; \mathbb{R}^{l}\right) \ni x(\cdot) \mapsto f(\cdot, x(\cdot)) \in L^{p_{2}}\left(\Omega ; \mathbb{R}^{m}\right)$ is continuous.

Let $S_{i}, i=1, \ldots, k$, be given sets of measurable functions on $\Omega$ with values in $\mathbb{R}^{h}$. Assume $L_{i} \subset S_{i}, i=1, \ldots, k$, are topological Hausdorff spaces such that any convergent sequence in $L_{i}$ contains a subsequence convergent (to the same limit) pointwise a.e. in $\Omega$. Put $L \subset L_{1} \times \cdots \times L_{k}$ with the topology induced from $L_{1} \times \cdots \times L_{k}$. Assume that $f: \Omega \times \mathbb{R}^{h_{1}} \times \cdots \times \mathbb{R}^{/ 4} \rightarrow \mathbb{R}^{m}$ is a Carathéodory function.

THEOREM 2. If, for any convergent sequence $\left(x_{n}^{1}, \ldots, x_{n}^{k}\right)_{n \in \mathbb{N}}$ in $L$ there exist a subsequence $\left(x_{n_{i}}^{1}, \ldots, x_{n_{i}}^{k}\right)_{i \in \mathrm{N}}$ and a function $h \in L^{p}\left(\Omega ; \mathbb{R}_{0}^{+}\right), 1 \leq p<\infty$, such that

$$
\left|f\left(t, x_{n_{i}}^{1}(t), \ldots, x_{n_{i}}^{k}(t)\right)\right| \leq h(t) \text { for all } i \in \mathbb{N} \text { and } t \in \Omega \text { a.e., }
$$

then the Nemytskii operator

$$
F: L \ni\left(x^{1}, \ldots, x^{k}\right) \mapsto f\left(\cdot, x^{1}(\cdot), \ldots, x^{k}(\cdot)\right) \in L^{p}\left(\Omega ; \mathbb{R}^{m}\right)
$$

is well defined and sequentially continuous in $L$, that is, if $\left(x_{n}^{1}, \ldots, x_{n}^{k}\right) \underset{n \rightarrow \infty}{\longrightarrow}\left(x_{0}^{1}, \ldots\right.$, $\left.x_{0}^{k}\right)$ in $L$, then $F\left(x_{n}^{1}, \ldots, x_{n}^{k}\right) \underset{n \rightarrow \infty}{\longrightarrow} F\left(x_{0}^{1}, \ldots, x_{0}^{k}\right)$ in $L^{p}\left(\Omega ; \mathbb{R}^{m}\right)$.

PROOF. The assumption of the theorem, applied to a constant sequence, implies that $F$ is well defined. Suppose now that $\left(x_{n}^{1}, \ldots, x_{n}^{k}\right) \underset{n \rightarrow \infty}{\longrightarrow}\left(x_{0}^{1}, \ldots, x_{0}^{k}\right)$ in $L$ and there exist a number $\varepsilon>0$ and a subsequence still denoted by $\left(x_{n}^{1}, \ldots, x_{n}^{k}\right)_{n \in \mathrm{N}}$, such that

$$
\int_{\Omega}\left|f\left(t, x_{n}^{1}(t), \ldots, x_{n}^{k}(t)\right)-f\left(t, x_{0}^{1}(t), \ldots, x_{0}^{k}(t)\right)\right|^{p} d t>\varepsilon \quad \text { for } n \in \mathbb{N} .
$$

On the other hand, we can choose a subsequence $\left(x_{n_{i}}^{1}, \ldots, x_{n_{i}}^{k}\right)$ of $\left(x_{n}^{1}, \ldots, x_{n}^{k}\right)$ satisfying (2.1). We calculate

$$
\begin{aligned}
\mid f(t, & \left.x_{n_{i}}^{1}(t), \ldots, x_{n_{i}}^{k}(t)\right)-\left.f\left(t, x_{0}^{1}(t), \ldots, x_{0}^{k}(t)\right)\right|^{p} \\
& \leq 2^{p}\left(\left|f\left(t, x_{n_{i}}^{1}(t), \ldots, x_{n_{i}}^{k}(t)\right)\right|^{p}+\left|f\left(t, x_{0}^{1}(t), \ldots, x_{0}^{k}(t)\right)\right|^{p}\right) \\
& \leq 2^{p}\left((h(t))^{p}+\left|f\left(t, x_{0}^{1}(t), \ldots, x_{0}^{k}(t)\right)\right|^{p}\right)
\end{aligned}
$$


for all $i \in \mathbb{N}$ and $t \in \Omega$ a.e. Since the topologies in $L_{j}, j=1, \ldots, k$, imply a pointwise convergence a.e. of a subsequence, the Carathéodory condition on $f$ implies that, up to a subsequence, $f\left(t, x_{n_{i}}^{1}(t), \ldots, x_{n_{j}}^{k}(t)\right)$ is pointwise convergent a.e. in $\Omega$ to $f\left(t, x_{0}^{1}(t), \ldots, x_{0}^{k}(t)\right)$. Using the Lebesgue dominated convergence theorem, we get $\lim _{i \rightarrow \infty} \int_{\Omega}\left|f\left(t, x_{n_{i}}^{1}(t), \ldots, x_{n_{i}}^{k}(t)\right)-f\left(t, x_{0}^{1}(t), \ldots, x_{0}^{k}(t)\right)\right|^{p} d t=0$, which contradicts (2.2) and the proof is completed.

REMARK. In the above theorem any subsets of $L^{p_{i}}\left(\Omega ; \mathbb{R}^{l_{l}}\right)$ or $C\left(\Omega, \mathbb{R}^{l_{l}}\right)$ with $\Omega$ compact can be considered as topological spaces $L_{i}$ (with the induced topologies).

\section{Application}

Let us consider a second order system

$$
\left\{\begin{array}{l}
\frac{d}{d t}\left(\dot{u}_{1}(t)+\alpha_{1}(t)\right)=-a u_{1}(t)+\beta_{1}(t)+A(t) u_{2}(t), \\
\frac{d}{d t}\left(\left|\dot{u}_{2}(t)\right|^{2} \dot{u}_{2}(t)+\alpha_{2}(t)\right)=-b\left|u_{2}(t)\right|^{2} u_{2}(t)+\beta_{2}(t)+A^{T}(t) u_{1}(t)
\end{array}\right.
$$

for $t \in I=[0, \pi]$ a.e., with Dirichlet boundary conditions $u(0)=u(\pi)=0$.

It is easy to notice that the functional of action for system (3.1) (which becomes the Euler-Lagrange equation for this functional) is of the form

$$
\begin{aligned}
\phi\left(u_{1}, u_{2}\right)= & \int_{l}\left(\frac{1}{2}\left|\dot{u}_{1}(t)\right|^{2}+\frac{1}{4}\left|\dot{u}_{2}(t)\right|^{4}+\left\langle\alpha_{1}(t), \dot{u}_{1}(t)\right\rangle\right. \\
& +\left\langle\alpha_{2}(t), \dot{u}_{2}(t)\right\rangle-\frac{a}{2}\left|u_{1}(t)\right|^{2}-\frac{b}{4}\left|u_{2}(t)\right|^{4}+\left\langle\beta_{1}(t), u_{1}(t)\right\rangle \\
& \left.+\left\langle\beta_{2}(t), u_{2}(t)\right\rangle+\left\langle u_{1}(t), A(t) u_{2}(t)\right\rangle\right) d t,
\end{aligned}
$$

where $a, b \in \mathbb{R}, \alpha_{1} \in L^{2}\left(I, \mathbb{R}^{l_{1}}\right) \backslash L^{4}\left(I, \mathbb{R}^{l_{1}}\right), \alpha_{2} \in L^{4 / 3}\left(I, \mathbb{R}^{l_{2}}\right), \beta_{1} \in L^{1}\left(I ; \mathbb{R}^{l_{1}}\right)$, $\beta_{2} \in L^{\prime}\left(I ; \mathbb{R}^{l_{2}}\right), A \in L^{\prime}\left(I ; \mathbb{R}^{l_{1} \times l_{2}}\right), l_{1}, l_{2} \in \mathbb{N}$. (Recall that $W_{0}^{1, p}\left(I, \mathbb{R}^{\prime}\right)$, where $p \in[1, \infty[, l \in \mathbb{N}$ is the standard Sobolev space, that is, the space of all absolutely continuous functions $u: l \mapsto \mathbb{R}^{l}$ such that $\dot{u} \in L^{p}\left(I, \mathbb{R}^{\prime}\right)$ and $u(0)=u(\pi)=0$, equipped with the norm $\left.\|u\|=\left(\int_{l}|u(t)|^{p} d t+\int_{I}|\dot{u}(t)|^{p} d t\right)^{1 / p}\right)$. At the first glance it seems that the space $W_{0}^{1.4}\left(l, \mathbb{R}^{1 / 1} \times \mathbb{R}^{1 / 2}\right)$ is a natural space for investigating system (3.1) as well as functional (3.2). However, from the fact that $\alpha_{1} \in L^{2}\left(I, \mathbb{R}^{\prime}\right) \backslash L^{4}\left(I, \mathbb{R}^{I_{1}}\right)$ it follows that there is no solution of system (3.1) in $W_{0}^{1,4}\left(I, \mathbb{R}^{l_{1}} \times \mathbb{R}^{l_{2}}\right)$. On the other hand, we easily check that $\phi$ is a Gâteaux-differentiable, weakly lower semicontinuous and coercive functional on $W_{0}^{1.2}\left(I, \mathbb{R}^{l_{1}}\right) \times W_{0}^{1.4}\left(I, \mathbb{R}^{l_{2}}\right)$. By applying a direct method of the calculus of variations it is easy to prove the existence of a solution of (3.1) 
in $W_{0}^{1,2}\left(I, \mathbb{R}^{1 /}\right) \times W_{0}^{1,4}\left(I, \mathbb{R}^{12}\right)$. In many variational and optimal control problems, an essential role is played by the theorem on the existence and continuity of the Fréchet derivative of integral functionals (see for example $[7,4]$ ). Using Theorem 2 , we can prove

THEOREM 3. Let I be a finite interval in $\mathbb{R}$, and let $g: I \times \mathbb{R}^{l_{1}} \times \mathbb{R}^{l_{2}} \times \mathbb{R}^{l_{1}} \times \mathbb{R}^{l_{2}} \rightarrow \mathbb{R}$ be a Carathéodory function continuously differentiable with respect to the last four variables for $t \in I$ a.e. If there exist functions $a_{1}, a_{2} \in C\left(I, \mathbb{R}_{0}^{+}\right), b \in L^{1}\left(I, \mathbb{R}_{0}^{+}\right)$, $c \in L^{q_{1}}\left(I, \mathbb{R}_{0}^{+}\right), d \in L^{q_{2}}\left(I, \mathbb{R}_{0}^{+}\right)$, where $p_{1}^{-1}+q_{1}^{-1}=1, p_{2}^{-1}+q_{2}^{-1}=1$, such that

$$
\begin{aligned}
\left|g\left(t, u_{1}, u_{2}, \dot{u}_{1}, \dot{u}_{2}\right)\right| & \leq\left(a_{1}\left(\left|u_{1}\right|\right)+a_{2}\left(\left|u_{2}\right|\right)\right)\left(b(t)+\left|\dot{u}_{1}\right|^{p_{1}}+\left|\dot{u}_{2}\right|^{p_{2}}\right), \\
\left|\nabla_{u_{1}} g\left(t, u_{1}, u_{2}, \dot{u}_{1}, \dot{u}_{2}\right)\right| & \leq\left(a_{1}\left(\left|u_{1}\right|\right)+a_{2}\left(\left|u_{2}\right|\right)\right)\left(b(t)+\left|\dot{u}_{1}\right|^{p_{1}}+\left|\dot{u}_{2}\right|^{p_{2}}\right), \\
\left|\nabla_{u_{2}} g\left(t, u_{1}, u_{2}, \dot{u}_{1}, \dot{u}_{2}\right)\right| & \leq\left(a_{1}\left(\left|u_{1}\right|\right)+a_{2}\left(\left|u_{2}\right|\right)\right)\left(b(t)+\left|\dot{u}_{1}\right|^{p_{1}}+\left|\dot{u}_{2}\right|^{p_{2}}\right), \\
\left|\nabla_{\dot{u}_{1}} g\left(t, u_{1}, u_{2}, \dot{u}_{1}, \dot{u}_{2}\right)\right| & \leq\left(a_{1}\left(\left|u_{1}\right|\right)+a_{2}\left(\left|u_{2}\right|\right)\right)\left(c(t)+\left|\dot{u}_{1}\right|^{p_{1}-1}+\left|\dot{u}_{2}\right|^{p_{2} / q_{1}}\right), \\
\left|\nabla_{\dot{u}_{2}} g\left(t, u_{1}, u_{2}, \dot{u}_{1}, \dot{u}_{2}\right)\right| & \leq\left(a_{1}\left(\left|u_{1}\right|\right)+a_{2}\left(\left|u_{2}\right|\right)\right)\left(d(t)+\left|\dot{u}_{2}\right|^{p_{2}-1}+\left|\dot{u}_{1}\right|^{p_{1} / q_{2}}\right),
\end{aligned}
$$

then the functional $\phi: W_{0}^{1, p_{1}}\left(I, \mathbb{R}^{l_{1}}\right) \times W_{0}^{1, p_{2}}\left(I, \mathbb{R}^{l_{2}}\right) \rightarrow \mathbb{B}$ defined by the formula

$$
\phi\left(u_{1}, u_{2}\right)=\int_{I} g\left(t, u_{1}(t), u_{2}(t), \dot{u}_{1}(t), \dot{u}_{2}(t)\right) d t
$$

is continuously Fréchet-differentiable in $W_{0}^{1, p_{1}}\left(I, \mathbb{R}^{l_{1}}\right) \times W_{0}^{1, p_{2}}\left(I, \mathbb{R}^{l_{2}}\right)$, and

$$
\begin{aligned}
\phi^{\prime}\left(u_{1}, u_{2}\right)\left(h_{1}, h_{2}\right)= & \int_{l}\left(\nabla_{u_{1}} g\left(t, u_{1}(t), u_{2}(t), \dot{u}_{1}(t), \dot{u}_{2}(t)\right) h_{1}(t)\right. \\
& +\nabla_{u_{2}} g\left(t, u_{1}(t), u_{2}(t), \dot{u}_{1}(t), \dot{u}_{2}(t)\right) h_{2}(t) \\
& +\nabla_{\dot{u}_{1}} g\left(t, u_{1}(t), u_{2}(t), \dot{u}_{1}(t), \dot{u}_{2}(t)\right) \dot{h}_{1}(t) \\
& \left.+\nabla_{\dot{u}_{2}} g\left(t, u_{1}(t), u_{2}(t), \dot{u}_{1}(t), \dot{u}_{2}(t)\right) \dot{h}_{2}(t)\right) d t
\end{aligned}
$$

for $\left(h_{1}, h_{2}\right) \in W_{0}^{1, p_{1}}\left(I, \mathbb{R}^{l_{1}}\right) \times W_{0}^{1, p_{2}}\left(I, \mathbb{R}^{l_{2}}\right)$.

REMARK. This theorem is a generalization of [7, Theorem 1.4] in the case of product of spaces $W_{0}^{1, p_{1}}\left(I, \mathbb{R}^{l_{1}}\right) \times W_{0}^{1, p_{2}}\left(I, \mathbb{R}^{l_{2}}\right)$. In a natural way it may be extended to the case of product of any finite number of spaces $W_{0}^{1, p_{i}}\left(I, \mathbb{B}^{l_{i}}\right)$.

In the proof of Theorem 3 we shall use this very interesting lemma of Brézis ([3, Theorem IV.9]).

LEMMA 1 (Brézis). From any sequence $\left(x_{n}\right)_{n \in \mathbb{N}}$ of elements of $L^{p}\left(\Omega ; \mathbb{R}^{l}\right), p \in$ $[1,+\infty)$, converging in $L^{p}\left(\Omega ; \mathbb{R}^{l}\right)$ to some $x_{0} \in L^{p}\left(\Omega ; \mathbb{R}^{l}\right)$, one can choose a subsequence $\left(x_{n_{k}}\right)_{k \in N}$ converging to $x_{0}$ a.e. in $\Omega$, such that $\left|x_{n_{k}}(t)\right| \leq g(t)$ for $t \in \Omega$ a.e. and $k \in \mathbb{N}$ with some function $g \in L^{p}\left(\Omega ; \mathbb{R}^{l}\right)$. 
PROOF OF THEOREM 3. It suffices to prove that there exist partial Gâteaux derivatives $\phi_{u_{1}}^{\prime}\left(u_{1}, u_{2}\right), \phi_{u_{2}}^{\prime}\left(u_{1}, u_{2}\right)$ of the functional $\phi$, continuous in $W_{0}^{1, p_{1}}\left(I, \mathbb{R}^{l_{1}}\right) \times$ $W_{0}^{1, p_{2}}\left(I, \mathbb{R}^{l_{2}}\right)$. It is easy to show (see for example [7, proof of Theorem 1.4]) that these derivatives exist and are given by the formulae

$$
\begin{aligned}
\phi_{u_{1}}^{\prime}\left(u_{1}, u_{2}\right)\left(h_{1}\right)= & \int_{I}\left(\nabla_{u_{1}} g\left(t, u_{1}(t), u_{2}(t), \dot{u}_{1}(t), \dot{u}_{2}(t)\right) h_{1}(t)\right. \\
& \left.+\nabla_{\dot{u}_{1}} g\left(t, u_{1}(t), u_{2}(t), \dot{u}_{1}(t), \dot{u}_{2}(t)\right) \dot{h}_{1}(t)\right) d t
\end{aligned}
$$

for $h_{1} \in W_{0}^{1, p_{1}}\left(I, \mathbb{R}^{l_{1}}\right)$ and

$$
\begin{aligned}
\phi_{u_{2}}^{\prime}\left(u_{1}, u_{2}\right)\left(h_{2}\right)= & \int_{I}\left(\nabla_{u_{2}} g\left(t, u_{1}(t), u_{2}(t), \dot{u}_{1}(t), \dot{u}_{2}(t)\right) h_{2}(t)\right. \\
& \left.+\nabla_{\dot{u}_{2}} g\left(t, u_{1}(t), u_{2}(t), \dot{u}_{1}(t), \dot{u}_{2}(t)\right) \dot{h}_{2}(t)\right) d t
\end{aligned}
$$

for $h_{2} \in W_{0}^{1, p_{2}}\left(I, \mathbb{R}^{l_{2}}\right)$. To end the proof, we must show that the operators

$$
\begin{aligned}
& W_{0}^{1, p_{1}}\left(I, \mathbb{R}^{l_{1}}\right) \times W_{0}^{1, p_{2}}\left(I, \mathbb{R}^{l_{2}}\right) \\
& \quad \ni\left(u_{1}, u_{2}\right) \mapsto \nabla_{u_{1}} g\left(\cdot, u_{1}(\cdot), u_{2}(\cdot), \dot{u}_{1}(\cdot), \dot{u}_{2}(\cdot)\right) \in L^{1}\left(I, \mathbb{R}^{l_{1}}\right)
\end{aligned}
$$

and

$$
\begin{aligned}
& W_{0}^{1, p_{1}}\left(I, \mathbb{R}^{l_{1}}\right) \times W_{0}^{1, p_{2}}\left(I, \mathbb{R}^{h_{2}}\right) \\
& \quad \ni\left(u_{1}, u_{2}\right) \mapsto \nabla_{\dot{u}_{1}} g\left(\cdot, u_{1}(\cdot), u_{2}(\cdot), \dot{u}_{1}(\cdot), \dot{u}_{2}(\cdot)\right) \in L^{1}\left(I, \mathbb{R}^{l_{1}}\right)
\end{aligned}
$$

for $i=1,2$ are continuous. Since $W_{0}^{1, p_{1}}\left(I, \mathbb{R}^{l_{1}}\right), W_{0}^{1, p_{2}}\left(I, \mathbb{R}^{l_{2}}\right)$ are metric spaces, it is enough to show that the above operators are sequentially continuous. Since the convergence of a sequence of functions in $W_{0}^{1, p_{1}}\left(I, \mathbb{R}^{l^{l}}\right)$ implies the convergence of this sequence in $C\left(I, \mathbb{R}^{l^{i}}\right)$ together with the convergence of the sequence of derivatives in $L^{p_{1}}\left(I, \mathbb{R}^{l_{1}}\right)$, therefore, by Theorem 2 , putting $L_{1}=C\left(I, \mathbb{R}^{l_{1}}\right), L_{2}=C\left(I, \mathbb{R}^{l_{2}}\right)$, $L_{3}=L^{p_{1}}\left(I, \mathbb{R}^{l_{1}}\right), L_{4}=L^{p_{2}}\left(I, \mathbb{R}^{l_{2}}\right)$ and $L=L_{1} \times L_{2} \times L_{3} \times L_{4}$, we get the assertion.

Indeed, let $\left(z_{1}^{n}, z_{2}^{n}, z_{3}^{n}, z_{4}^{n}\right) \underset{n \rightarrow \infty}{\longrightarrow}\left(z_{1}^{0}, z_{2}^{0}, z_{3}^{0}, z_{4}^{0}\right)$ in $L$. Applying Lemma 1 , one can choose subsequences $\left(z_{3}^{n_{i}}\right)_{i \in \mathrm{N}}$ and $\left(z_{4}^{n_{i}}\right)_{i \in \mathrm{N}}$ such that $\left|\left(z_{3}^{n_{1}}(t)\right)\right| \leq g_{3}(t)$ and $\left|\left(z_{4}^{n_{i}}(t)\right)\right| \leq$ $g_{4}(t)(t \in I$ a.e., $i \in \mathbb{N})$ for some functions $g_{3} \in L^{p_{1}}\left(I, \mathbb{R}_{0}^{+}\right)$and $g_{4} \in L^{p_{2}}\left(I, \mathbb{R}_{0}^{+}\right)$. Moreover, since $L_{1}=C\left(I, \mathbb{R}^{l_{1}}\right), L_{2}=C\left(I, \mathbb{R}^{l_{2}}\right)$, there exists $M \geq 0$ such that

$$
\left|\left(z_{1}^{n_{i}}(t)\right)\right| \leq M \quad \text { and } \quad\left|\left(z_{2}^{n_{i}}(t)\right)\right| \leq M, \quad t \in I, i \in \mathbb{N} .
$$

Hence the assumptions of the theorem yield, for $j=1,2$, the estimates

$$
\left|\nabla_{u_{j}} g\left(t, z_{1}^{n_{i}}, z_{2}^{n_{i}}, z_{3}^{n_{i}}, z_{4}^{n_{i}}\right)\right| \leq h_{j}(t), \quad t \in I \text { a.e., } i \in \mathbb{N},
$$

with $h_{j}(t)=\left(a_{1}(M)+a_{2}(M)\right)\left(b(t)+\left|g_{3}(t)\right|^{p_{1}}+\left|g_{4}(t)\right|^{p_{2}}\right)$ belonging to $L_{1}\left(I, \mathbb{R}_{0}^{+}\right)$. 
In the same way, for $j=1,2$, one can check that

$$
\left|\nabla_{\dot{u}_{j}} g\left(t, z_{1}^{n_{i}}, z_{2}^{n_{i}}, z_{3}^{n_{i}}, z_{4}^{n_{i}}\right)\right| \leq \bar{h}_{j}(t), \quad t \in l \text { a.e., } i \in \mathbb{N},
$$

with $\bar{h}_{1} \in L^{q_{1}}\left(I, \mathbb{R}_{0}^{+}\right), \bar{h}_{2} \in L^{q_{2}}\left(I, \mathbb{R}_{0}^{+}\right)$.

Consequently, each of the functions $\nabla_{u_{j}} g, \nabla_{\dot{u}_{j}} g, j=1,2$, satisfies the assumptions of Theorem 2. Therefore we conclude that if $\left(u_{1}^{n}, u_{2}^{n}\right) \underset{n \rightarrow \infty}{\longrightarrow}\left(u_{1}^{0}, u_{2}^{0}\right)$ in $W_{0}^{1, p_{1}}\left(I, \mathbb{R}^{l_{1}}\right) \times$ $W_{0}^{1, p_{2}}\left(I, \mathbb{R}^{l_{2}}\right)$, then

$$
\nabla_{u j} g\left(\cdot, u_{1}^{n}(\cdot), u_{2}^{n}(\cdot), \dot{u}_{1}^{n}(\cdot), \dot{u}_{2}^{n}(\cdot)\right) \underset{n \rightarrow \infty}{\longrightarrow} \nabla_{u j} g\left(\cdot, u_{1}^{0}(\cdot), u_{2}^{0}(\cdot), \dot{u}_{1}^{0}(\cdot), \dot{u}_{2}^{0}(\cdot)\right)
$$

in $L^{1}\left(l, \mathbb{R}^{l}\right)$ for $j=1,2$, and

$$
\nabla_{\dot{u} j} g\left(\cdot, u_{1}^{n}(\cdot), u_{2}^{n}(\cdot), \dot{u}_{1}^{n}(\cdot), \dot{u}_{2}^{n}(\cdot)\right) \underset{n \rightarrow \infty}{\longrightarrow} \nabla_{\dot{u}_{j}} g\left(\cdot, u_{1}^{0}(\cdot), u_{2}^{0}(\cdot), \dot{u}_{1}^{0}(\cdot), \dot{u}_{2}^{0}(\cdot)\right)
$$

in $L^{q_{j}}\left(l, \mathbb{R}^{l_{j}}\right)$ for $j=1,2$. This ends the proof.

\section{References}

[1] J. Appell and P. P. Zabrejko, 'Boundedness properties of the superposition operator', Bull. Polish Acad. Sci. Math. 37 (1989), 363-377.

[2] — 'Continuity properties of the superposition operator', J. Austral. Math. Soc. Ser. A 47 (1989), 186-210.

[3] H. Brézis, Analyse fonctionelle. Théorie et applications (Masson, Paris, 1983).

[4] A. Ioffe and V. Tikhomirov, Theory of extremal problems (North-Holland, Amsterdam, 1979).

[5] M. K. Krasnoselskii, Topological methods in the theory of nonlinear integral equations (Pergamon Press, New York, 1964).

[6] M. K. Krasnoselskii, P. P. Zabreiko, J. I. Pustylnik and P. J. Sobolevskii, Integral operators in spaces of summable functions (Noordhoff, Leyden, 1976).

[7] J. Mawhin and P. Willem, Critical point theory and Hamiltonian systems (Springer, New York, 1989).

[8] M. Struwe, Variational methods (Springer, Berlin, 1990).

[9] M. M. Vainberg, Variational method and method of monotone operators in the theory of nonlinear equations (Halsted Press, New York, 1973).

[10] M. Willem, Minimax theorems (Birkhäuser, Basel, 1996).

Faculty of Mathematics

University of Łódź

S. Banacha 22

90-238 Łódź, Poland

e-mail: idczak@math.uni.lodz.pl, arogow@math.uni.lodz.pl 